\title{
Factors affecting the ability of abdominal ultrasonography to detect focal pancreatic lesions identified using endoscopic ultrasonography
}

\author{
Seo-Youn Choi ${ }^{1}$, Jung Hoon $\mathrm{Kim}^{2,3}$, Hyo Won Eun ${ }^{4}$, Hwaseong Ryu ${ }^{5}$ \\ 'Department of Radiology, Soonchunhyang University Bucheon Hospital, Bucheon; \\ ${ }^{2}$ Department of Radiology, Seoul National University Hospital, Seoul; ${ }^{3}$ Institute of Radiation \\ Medicine, Seoul National University College of Medicine, Seoul; ${ }^{4}$ Department of Radiology, \\ Samsung Medical Center, Seoul; ${ }^{5}$ Department of Radiology, Pusan National University \\ Yangsan Hospital, Yangsan, Korea
}

Purpose: This study was conducted to determine which factors influence the ability of abdominal ultrasonography (US) to detect focal pancreatic lesions identified using endoscopic ultrasonography (EUS).

Methods: In this study, 338 consecutive patients with focal pancreatic lesions (cyst, $n=253$; adenocarcinoma, $n=54$; pancreatic neuroendocrine tumor, $n=24$; solid pseudopapillary neoplasm, $n=4$; intrapancreatic accessory spleen, $n=1$; metastasis, $n=1$; and lymphoma, $n=1$ ) detected by EUS who underwent US were enrolled. We reviewed their radiologic reports and assessed the presence or absence of a focal lesion, the multiplicity of the lesions, and their size and location on US. We evaluated how these parameters differed depending on whether the lesion was solid or cystic. Univariate and multivariate logistic regression analysis were performed. Results: The overall detection rate of focal pancreatic lesions by US was $61.5 \%$ (208 of 338). Using US, the detection rate of cystic lesions was $58.5 \%$ (148 of 253), while that of solid lesions was $70.6 \%$ (60 of 85 ). In the univariate analysis, location in the neck or body, solid characteristics, and a relatively large size $(15.50 \pm 10.08 \mathrm{~mm}$ vs. $23.09 \pm 12.93 \mathrm{~mm})$ were associated with a significantly higher detection rate $(P<0.001, P=0.047$, and $P<0.001$, respectively). In the multivariate analysis, location in the neck or body (odds ratio [OR], 3.238; 95\% confidence interval $[\mathrm{Cl}], 1.926$ to $5.443 ; \mathrm{P}<0.001)$ and size $(\mathrm{OR}, 1.070 ; 95 \% \mathrm{Cl}, 1.044$ to 1.096; $\mathrm{P}<0.001)$ were proven to be significant predictors of detectability $(\mathrm{P}<0.001)$.

Conclusion: US is useful for detecting focal pancreatic lesions, especially when they are located in the neck or body and are relatively large.

Keywords: Pancreas; Ultrasonography; Neoplasms; Cysts; Endoscopy

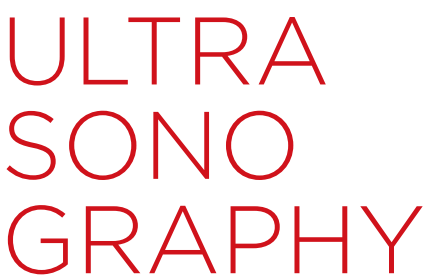

ORIGINAL ARTICLE

https://doi.org/10.14366/usg.19078 pISSN: 2288-5919 • elSSN: 2288-5943 Ultrasonography 2020;39:247-256

Received: November 19, 2019

Revised: February 7, 2020

Accepted: February 9, 2020

Correspondence to: Jung Hoon Kim, MD, Department of Radiology, Seoul National University Hospital, 101 Daehak-ro, Jongno-gu, Seoul 03080, Korea

Tel. +82-2-2072-1969

Fax. +82-2-743-6385

E-mail: jhkim2008@gmail.com

This is an Open Access article distributed under the terms of the Creative Commons Attribution NonCommercial License (http://creativecommons.org/ licenses/by-nc/4.0/) which permits unrestricted noncommercial use, distribution, and reproduction in any medium, provided the original work is properly cited.

Copyright $(2) 2020$ Korean Society of Ultrasound in Medicine (KSUM)

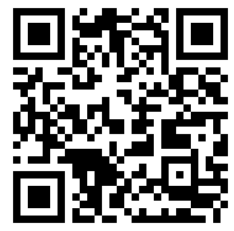

How to cite this article:

Choi SY, Kim JH, Eun HW, Ryu H. Factors affecting the ability of abdominal ultrasonography to detect focal pancreatic lesions identified using endoscopic ultrasonography. Ultrasonography. 2020 Jul;39(3):247-256. 


\section{Introduction}

The number of incidentally discovered pancreatic lesions has increased with advances in the resolution of cross-sectional imaging. The prevalence of incidental pancreatic cystic lesions has been reported to be up to $2.6 \%$ using computed tomography (CT) and up to $20 \%$ using magnetic resonance imaging (MRI) [1-5]. Although the majority of pancreatic cystic lesions are considered to be premalignant, the current guidelines suggest follow-up of pancreatic cysts, especially for cysts smaller than $3 \mathrm{~cm}$ [6]. However, the malignancy risk of cystic lesions less than $3 \mathrm{~cm}$ was reported to be $1 \%$ to $3 \%[7,8]$, and it is also known that patients with pancreatic cysts are at an increased risk of pancreatic cancer elsewhere in the pancreas $[9,10]$. Pancreatic cancer is by far the most common primary solid tumor of the pancreas, and it remains one of the leading causes of cancer-related death, with a dismal prognosis [11].

Conventional abdominal ultrasonography (US) is the imaging modality of choice for the first-line evaluation of a patient with abdominal disease. US has the advantages of noninvasiveness, costeffectiveness, wide availability, and easy accessibility. However, US has limitations for the pancreas due to its poor sonic window, which results from the deep retroperitoneal location of the pancreas, as well as disruption of the transmission of US waves by bowel gas. The pancreas may also be difficult to visualize depending on the patient's body habitus, and visualizing the pancreas depends heavily on the operator's skill. For this reason, the diagnostic performance of US for detecting pancreatic focal lesions has been reported to be lower than that of other imaging modalities, including CT, MRI, and endoscopic US (EUS), although only a few reports have been published regarding pancreatic lesion evaluation using US [1215]. The technical success of US obviously depends on the skill and perseverance of the examiner [16]. Furthermore, factors related to the patient or the lesion itself can also affect the results of US. However, to the best of our knowledge, few studies have sought to identify the factors affecting the diagnostic performance of US for pancreatic focal lesions, and no reports whatsoever have attempted to determine the most significant determinants.

EUS is recognized as the best imaging modality for focal pancreatic solid or cystic lesions, and has been suggested to be valuable as a problem-solving tool due to its high spatial resolution $[6,17,18]$. In addition, the sensitivity of EUS for pancreatic tumors has been reported to be $90 \%$ to $100 \%[19,20]$. Although it is widely used for its interventional advantages, such as for fine-needle aspiration cytology or biopsy, EUS is a more invasive technique than conventional US. In addition, if a pancreatic cystic lesion is well visualized on US, US could play an important role in the follow-up management of the lesion, potentially replacing other cross-sectional imaging modalities such as CT and MRI [21-23]. Therefore, the purpose of this study was to determine which factors influence the detectability of focal pancreatic lesions by US.

\section{Materials and Methods}

\section{Patient Population}

This retrospective study was approved by our institutional review board, and the requirement to obtain informed consent from patients was waived. We reviewed the medical, pathology, and radiology databases of our institution from January 2014 through September 2015, and we found 508 consecutive patients who visited our institution and underwent EUS under suspicion of a pancreatic abnormality based on previous $\mathrm{CT}$ or MRI findings. We excluded 120 of those patients who did not receive abdominal US, regardless of the temporal relationship between EUS and abdominal US. Fifty other patients were excluded for the following reasons: no pancreatic focal lesion other than pancreatitis $(n=19)$, main pancreatic duct (MPD) dilatation without a focal lesion $(n=17)$, peripancreatic fat invagination $(n=9)$, normal EUS findings $(n=4)$, and diffuse lymphoma $(n=1)$. Finally, 338 consecutive patients (153 males and 185 females; mean age, 62.73 years; range, 15 to 87 years; 253 with cystic lesions and 85 with solid lesions of the pancreas) were included in this study. When a patient had two or more lesions, the largest lesion was evaluated. The patients' age and multiplicity were used as qualitative parameters in analysis. Cystic lesions of the pancreas were defined as anechoic lesions with an imperceptibly thin wall and no solid nodule/component. Of the 253 cystic lesions, 39 lesions were pathologically confirmed through surgery ( $n=24)$ or biopsy ( $n=15)$ using US or EUS. The solid pancreatic lesions included adenocarcinoma $(n=54)$, neuroendocrine tumor $(n=24)$, solid pseudopapillary tumor $(n=4)$, intrapancreatic accessory spleen $(n=1)$, metastasis $(n=1)$, and focal-type lymphoma $(n=1)$. All of the solid lesions were pathologically confirmed by surgery or biopsy. The mean time interval between EUS and US was 6.58 months (range, 0 days to 26 months). Fig. 1 presents the flowchart of this study.

\section{EUS Examinations}

For the EUS procedure, under conscious sedation with 3 to $5 \mathrm{mg}$ of midazolam, patients were prepared with local pharyngeal anesthesia using a $2 \%$ lidocaine spray. The EUS examination was done by an gastroenterologist with more than 10 years of EUS experience, and the entire pancreas was assessed using a radial echoendoscope (GFUE 240, Olympus Co., Tokyo, Japan; Aloka SSD-alpha 10 Ultrasound System, Aloka Co., Ltd., Tokyo, Japan) with a 7.5 to $12 \mathrm{MHz}$ rotating transducer (GF-UM2, -UM3, -UM20, Olympus Co.). The endoscope 
was introduced in the left lateral decubitus position through the stomach and moved further down into the duodenal bulb or the second portion of the duodenum. The pancreas was assessed during this procedure. After the EUS examination, the final results were recorded in a structured reporting format.

\section{Abdominal US Examinations}

For abdominal US, it was necessary for all patients to fast for more than 6 hours in order to improve the visibility of the pancreas and to reduce the amount of bowel gas. US was performed using one of the following commercially available US units: Aplio XG (Toshiba Medical Systems, Otawara, Japan), Logiq 9 (GE Healthcare, Milwaukee, WI, USA), iU-22 (Philips Medical Systems, Bothell, WA, USA), ACUSON S2000 (Siemens Healthcare, Erlangen, Germany), or Aixplorer (Supersonic Imagine, Aix-en-Provence, France). For each patient, US was performed by one of the assigned radiologists with a range of 2 to 35 years of clinical experience in abdominal US. Before starting the examination, we carefully reviewed each patient's past clinical history, chief complaint, and previously obtained images. During the US procedure, the pancreas was carefully investigated through the subcostal approach with the patient in a supine position, using a convex, low-frequency transducer (bandwidth, 1.5-4.5 MHz) with and without harmonic imaging or color Doppler US. The US parameters were as follows: frequency, $4 \mathrm{MHz}$; gain, $27 \%$ to $33 \%$; dynamic range, 69; and frame rate, 30 to 45 pictures per second. As part of a careful evaluation of the pancreas, we applied different scanning techniques, including transverse scanning and oblique scanning with breathing control and with suspended inspiration or expiration, changing the patient's position to the supine and left decubitus positions. If required, we also used compression to displace bowel gas. Harmonic imaging was usually used. Color Doppler studies were also performed in most cases depending on the operator's need to assess the vascularity of the pancreatic focal lesion. For evaluation of the pancreatic tail, we used the spleen as a sonic window through the left intercostal space.

508 Consecutive patients underwent EUS under suspicion of pancreatic abnormality from January 2014 to September 2015

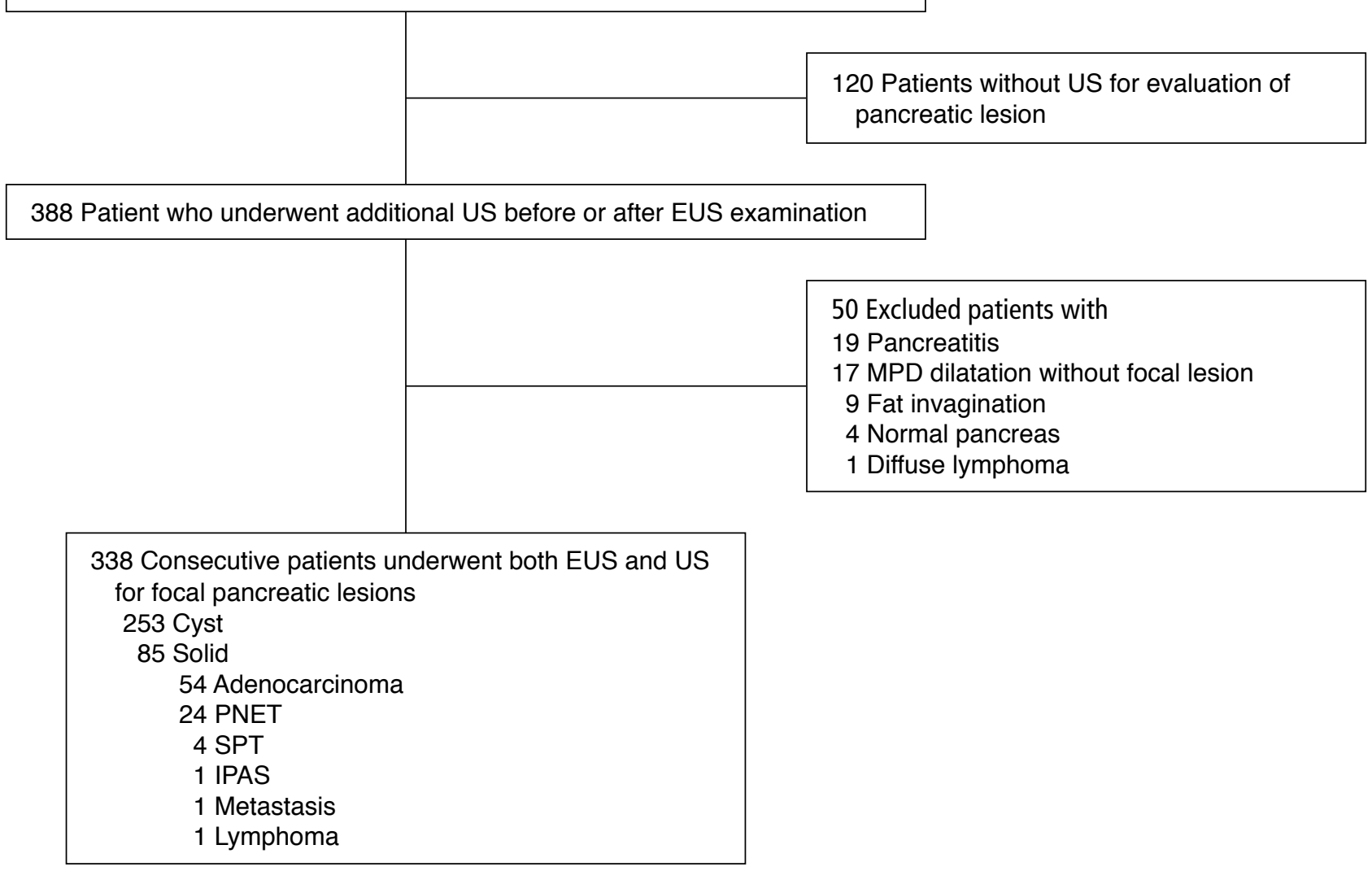

Fig. 1. Flow chart of study subjects. EUS, endoscopic ultrasonography; US, abdominal ultrasonography; MPD, main pancreatic duct; PNET, pancreatic neuroendocrine tumor; SPT, solid pseudopapillary tumor; IPAS, intrapancreatic accessory spleen. 


\section{Image Interpretation}

Two board-certified radiologists with 21 and 10 years, respectively, of clinical experience in abdominal sonography (J.H.K. and S.Y.C.), retrospectively analyzed the sonograms based on the formal reports focusing on the presence of focal pancreatic lesions. All images were reviewed on a picture archiving and communication system workstation (INFINITT PACS, Infinitt Healthcare Co. Ltd., Seoul, Korea). We also analyzed the multiplicity of focal pancreatic lesions (single or multiple), whether the lesions had solid or cystic characteristics, and the size and location of the focal lesions. The location of a focal pancreatic lesion was classified as uncinate process/head, neck/body, or tail, based on the following definitions:

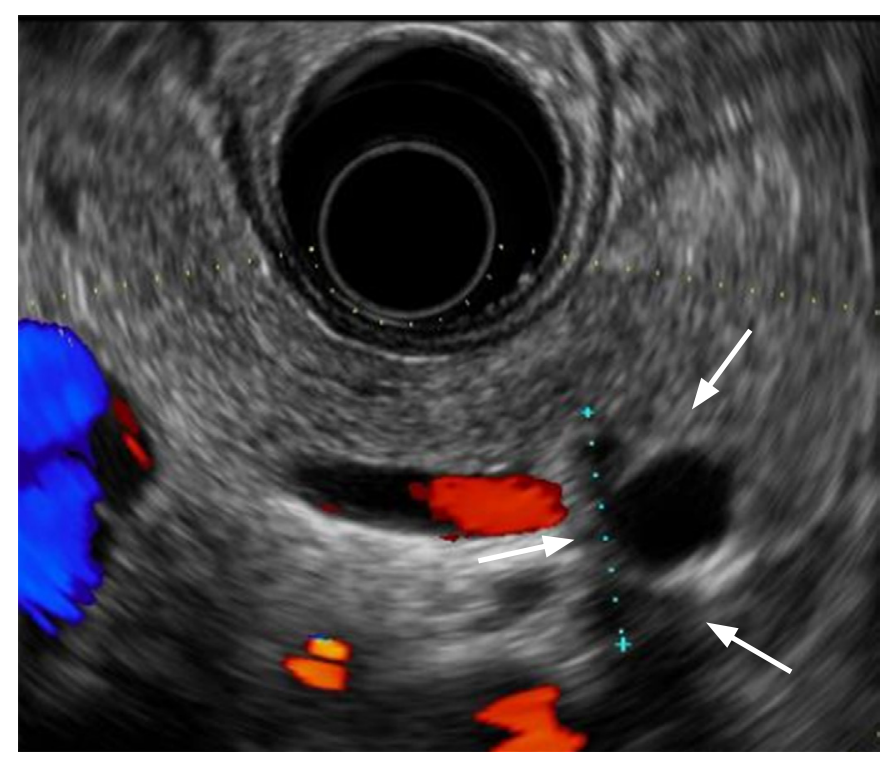

A

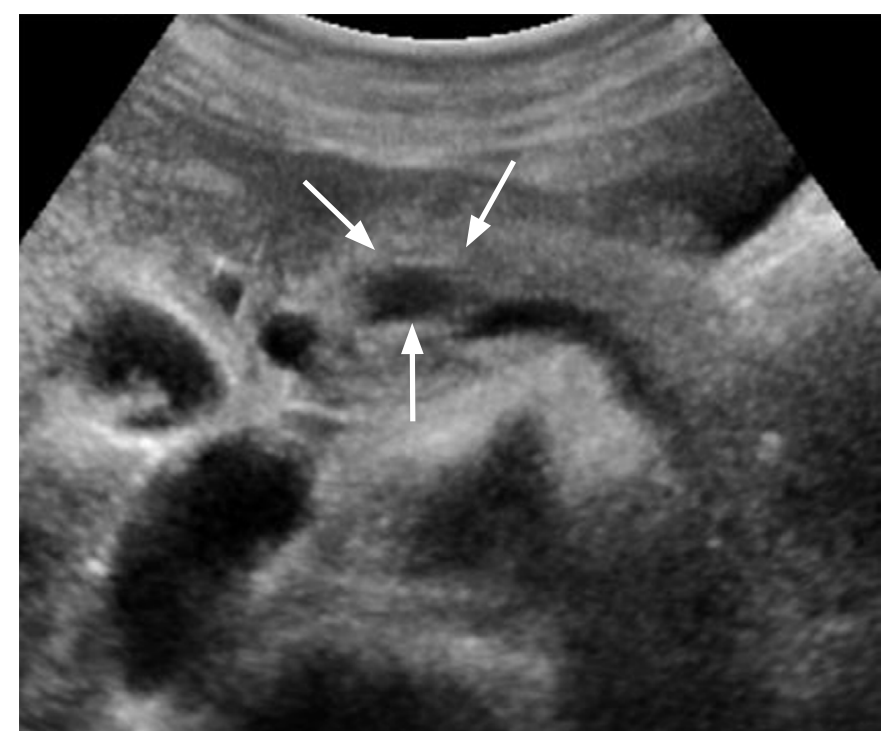

C head, right of the superior mesenteric vein (SMV); uncinate process, extension of the head and posterior to the SMV; neck, anterior to the SMV; body, left of the SMV and right of the left lateral border of the vertebral body; and tail, left of the left lateral border of the vertebral body to the splenic hilum. When the description on the formal report was unclear, we made the final decision by consensus.

\section{Statistical Analysis}

The statistical significance of differences in lesion characteristics between those that were detected and not detected by US were evaluated by means of the chi-square test for categorical variables and the Student t-test for continuous variables, as appropriate.

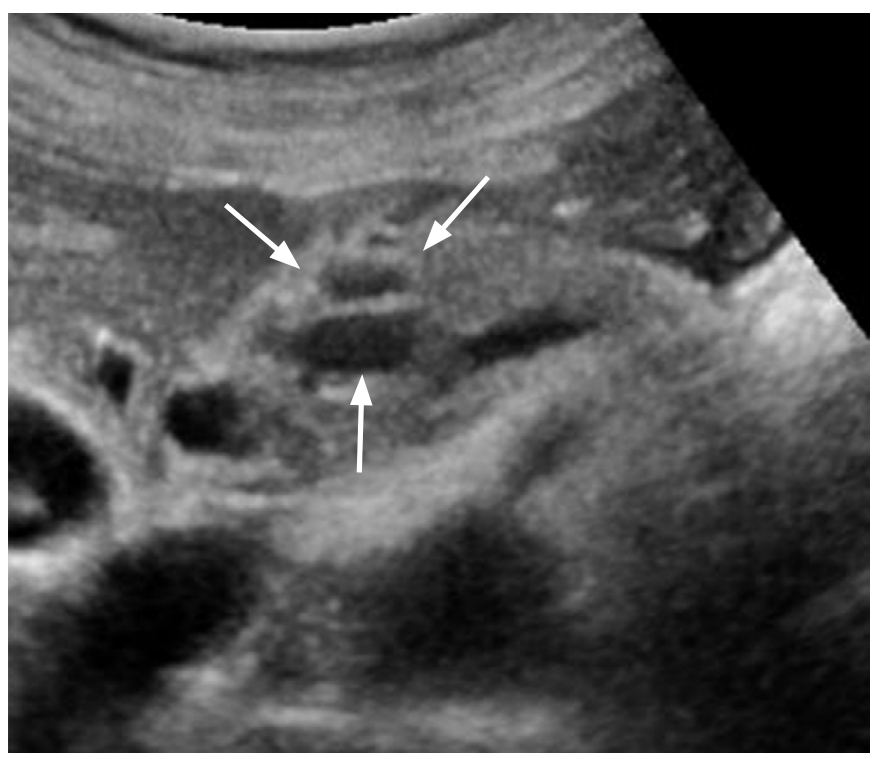

B

Fig. 2. Pancreatic cyst detected on abdominal ultrasonography (US) in a 49-year-old man.

A. Endoscopic US shows a 1.7-cm well-defined hypoechoic lesion in the body of the pancreas (arrows) with a thin septum. B, C. US also shows a 1.7-cm cystic lesion in the body of the pancreas (arrows) with a thin septum. 
To determine the factors affecting the ability of US to detect focal pancreatic lesions, stepwise multivariable logistic regression analyses were conducted using variables found to be significant in the univariate analysis. The area under the receiver operating characteristic (ROC) curve (AUC) and the 95\% confidence interval $(\mathrm{Cl})$ of the AUC was computed in order to evaluate the diagnostic ability of US for assessing the lesion size. The appropriate cut-off value of the lesion size, corresponding to the maximum Youden index, was estimated using ROC analysis. A 2-tailed P-value of $<0.05$ was considered to indicate statistical significance. All statistical analyses were performed using SPSS for Windows version 19.0 (IBM Corp., Armonk, NY, USA).

\section{Results}

Abdominal US detected 208 of the $338(61.5 \%)$ focal pancreatic lesions found on EUS (61.5\%). The ability of US to detect focal pancreatic lesions differed significantly depending on whether the lesions were located in the uncinate process and head $(89$ of $140,63.6 \%$ ), neck or body (94 of $124,75.8 \%$ ), or tail (25 of

Table 1. Demographic data of the enrolled patients and focal pancreatic lesions

\begin{tabular}{lc}
\hline \multicolumn{1}{c}{ Variable } & Value $(\mathrm{n}=338)$ \\
\hline Age $(\mathrm{yr})$ & $62.73 \pm 1.95(15-87)$ \\
Sex & $158(46.7)$ \\
Male & $180(53.3)$ \\
Female & $20.17 \pm 12.46(2.0-71.0)$ \\
Lesion size (mm) & \\
Lesion type & $85(25.1)$ \\
Solid & $253(74.9)$ \\
Cystic & \\
Detection on US & $208(61.5)$ \\
US detected & $130(38.5)$ \\
US non-detected & \\
Lesion number & $291(86.1)$ \\
Single & $47(13.9)$ \\
Multiple & \\
Location & $64(18.9)$ \\
Uncinate process & $76(22.5)$ \\
Head & $22(6.5)$ \\
Neck & $102(30.2)$ \\
Body & $74(21.9)$ \\
Tail & \\
\hline
\end{tabular}

Values are presented as mean \pm standard deviation (range) or number (\%). US, ultrasonography.
74, 33.8\%) $(P<0.001)$ (Fig. 2). The ability of US to detect solid focal pancreatic lesions was significantly higher than its ability to detect cystic focal pancreatic lesions (60 of $85,70.6 \%$ vs. 148 of $253,58.5 \% ; P=0.047)$. The lesion size also differed significantly between undetected and detected lesions $(15.50 \pm 10.08 \mathrm{~mm}$ vs. $23.09 \pm 12.93 \mathrm{~mm}$, respectively; $\mathrm{P}<0.001$ ) and between solid and cystic lesions $(27.65 \pm 14.57 \mathrm{~mm}$ vs. $17.66 \pm 10.56 \mathrm{~mm}$, respectively; $\mathrm{P}<0.001)$. However, multiplicity and patient age were not significant factors affecting the ability of US to detect lesions. Table 1 summarizes the demographic data and Table 2 summarizes the factors influencing the ability of US to detect focal pancreatic lesions.

The AUC of lesion size for the detectability of US was 0.695 ( $95 \% \mathrm{Cl}, 0.637$ to 0.752$)$. The optimal cut-off point of lesion size for determining its detectability by US was $17.5 \mathrm{~mm}$ in the ROC curve analysis (Fig. 3). By applying this cut-off value, the sensitivity and specificity were maximized (58.7\% and $68.5 \%$, respectively). Regardless of whether the lesion was cystic or solid, the ability of US to detect a lesion significantly depended on its location $(P<0.001$ for cystic lesions and $\mathrm{P}=0.001$ for solid lesions). However, while the detectability of cystic lesions by US was affected by the lesion size $(13.04 \pm 7.15 \mathrm{~mm}$ vs. $20.93 \pm 11.36 \mathrm{~mm}$, respectively; $\mathrm{P}<0.001)$, the size of undetected and detected solid lesions did not differ significantly $(P=0.448)$. In addition, the size of solid lesions was also significantly larger than that of cystic lesions $(17.66 \pm 10.56 \mathrm{~mm}$ vs.

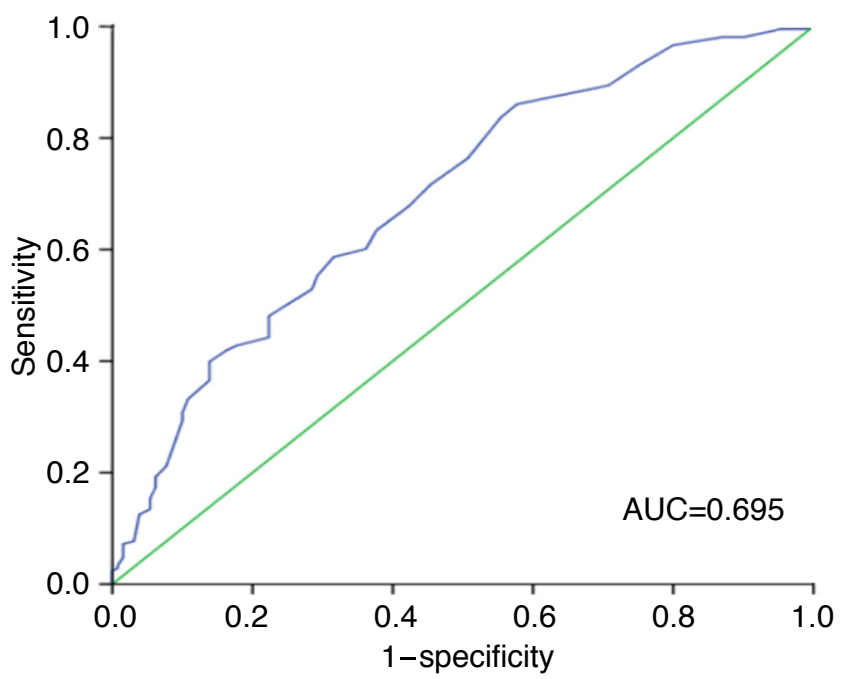

Fig. 3. Receiver operating characteristic curve analysis of the ability of abdominal ultrasonography to detect focal pancreatic lesions according to lesion size. The optimal cut-off value of lesion size for determining its detectability by ultrasonography was 17.5 $\mathrm{mm}$ (sensitivity of $58.7 \%$ and specificity of $68.5 \%$ ). AUC, area under the receiver operating characteristic curve. 
Table 2. Qualitative and quantitative factors influencing the ability of abdominal ultrasonography to detect focal pancreatic lesions

\begin{tabular}{|c|c|c|c|c|c|c|c|c|c|}
\hline \multirow[b]{2}{*}{ Variable } & \multicolumn{3}{|c|}{ All pancreatic lesions } & \multicolumn{3}{|c|}{ Cystic lesions } & \multicolumn{3}{|c|}{ Solid lesions } \\
\hline & $\begin{array}{l}\text { Undetected } \\
\qquad(n=130)\end{array}$ & $\begin{array}{l}\text { Detected } \\
(n=208)\end{array}$ & P-value & $\begin{array}{l}\text { Undetected } \\
(\mathrm{n}=105)\end{array}$ & $\begin{array}{c}\text { Detected } \\
(n=148)\end{array}$ & P-value & $\begin{array}{l}\text { Undetected } \\
\qquad(n=25)\end{array}$ & $\begin{array}{c}\text { Detected } \\
(n=60)\end{array}$ & P-value \\
\hline Characteristics & & & 0.047 & & & - & & & - \\
\hline Cystic & 105 & 148 & & - & - & & - & - & \\
\hline Solid & 25 & 60 & & - & - & & - & - & \\
\hline Multiplicity & & & 0.534 & & & 0.659 & & & NA \\
\hline Single & 110 & 181 & & 85 & 123 & & 25 & 58 & \\
\hline Multiple & 20 & 27 & & 20 & 25 & & - & 2 & \\
\hline Location & & & $<0.001$ & & & $<0.001$ & & & 0.001 \\
\hline Uncinate process/head & 51 & 89 & & 42 & 62 & & 9 & 27 & \\
\hline Neck/body & 30 & 94 & & 25 & 67 & & 5 & 27 & \\
\hline Tail & 49 & 25 & & 38 & 19 & & 11 & 6 & \\
\hline Size (mm) & $15.50 \pm 10.08$ & $23.09 \pm 12.93$ & $<0.001$ & $13.04 \pm 7.15$ & $20.93 \pm 11.36$ & $<0.001$ & $25.84 \pm 12.93$ & $28.40 \pm 14.99$ & 0.448 \\
\hline
\end{tabular}

Values are presented as number or mean \pm standard deviation.

NA, not available.

Table 3. Significant factors influencing the ability of abdominal ultrasonography to detect focal pancreatic lesions

\begin{tabular}{|c|c|c|c|c|}
\hline \multirow{2}{*}{ Variable } & \multicolumn{2}{|c|}{ Univariable } & \multicolumn{2}{|c|}{ Multivariable } \\
\hline & OR $(95 \% \mathrm{Cl})$ & P-value & OR $(95 \% \mathrm{Cl})$ & P-value \\
\hline Characteristics & & 0.047 & & - \\
\hline (ystic ${ }^{a)}$ & 1 & & & \\
\hline Solid & $1.703(1.003-2.891)$ & & - & \\
\hline Multiplicity & & 0.534 & & - \\
\hline Single $e^{a)}$ & 1 & & & \\
\hline Multiple & $1.219(0.652-2.277)$ & & - & \\
\hline Location & & $<0.001$ & & $<0.001$ \\
\hline Neck/body & $2.749(1.682-4.491)$ & & $3.238(1.926-5.443)$ & \\
\hline Others $^{\mathrm{a})}$ & 1 & & 1 & \\
\hline Size (mm) & $1.064(1.039-1.089)$ & $<0.001$ & $1.070(1.044-1.096)$ & $<0.001$ \\
\hline
\end{tabular}

$\mathrm{OR}$, odds ratio; $\mathrm{Cl}$, confidence interval.

Reference parameter.

$27.65 \pm 14.59 \mathrm{~mm}$, respectively; $\mathrm{P}<0.001$ ) (Fig. 4).

In the multivariate analysis, location of the lesion in the neck or body and its size were independently significant factors associated with the ability of US to detect focal pancreatic lesions. The detection rate of focal pancreatic lesions of the neck or body by US was significantly higher than the detection rate of lesions in other locations (odds ratio [OR], 3.238; $95 \% \mathrm{Cl}, 1.926$ to 5.443 ; $\mathrm{P}<0.001)$. Lesions located in the neck/body of the pancreas showed the highest detectability (76 of $102,74.5 \%$ ) compared to those in other parts of the pancreas (Fig. 5). In addition, the detectability of focal pancreatic lesions by US was significantly affected by the lesion size $(\mathrm{OR}, 1.070 ; 95 \% \mathrm{Cl}, 1.044$ to $1.096 ; \mathrm{P}<0.001)$. Table 3 summarizes the factors that significantly influenced the ability of US to detect focal pancreatic lesions using multivariate analysis. Although whether a focal pancreatic lesion was solid or cystic was significantly related with its detectability by US in the univariate analysis, it was not independently significant in the multivariate analysis.

\section{Discussion}

Our study demonstrated that the overall detection rate of focal pancreatic lesions by US was $61.5 \%$ (208 of 338), and the detection rate of pancreatic lesions in the neck/body was $74.5 \%$. Location 


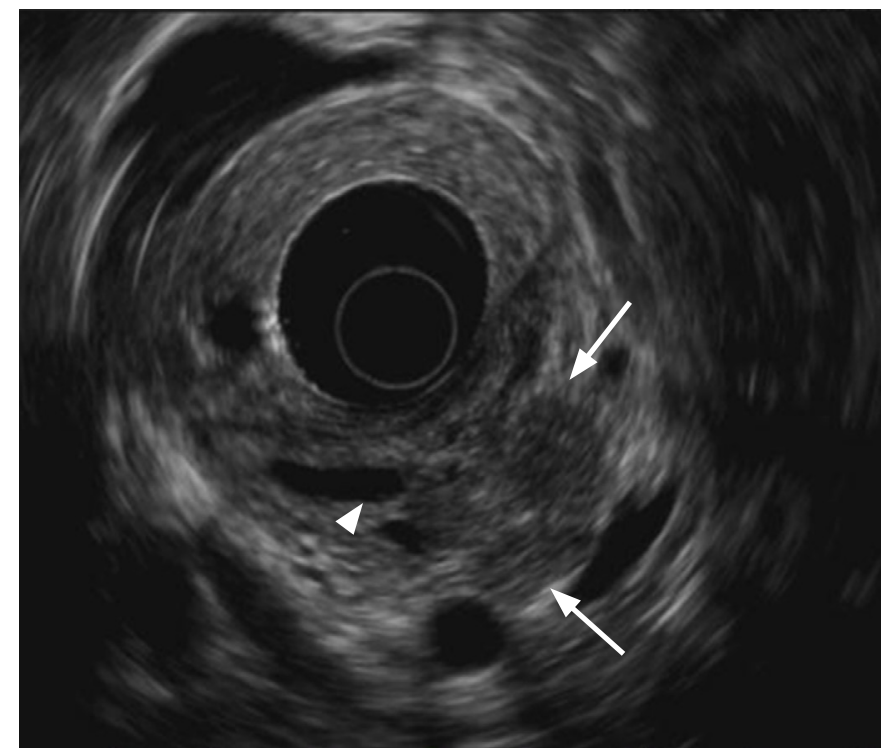

A

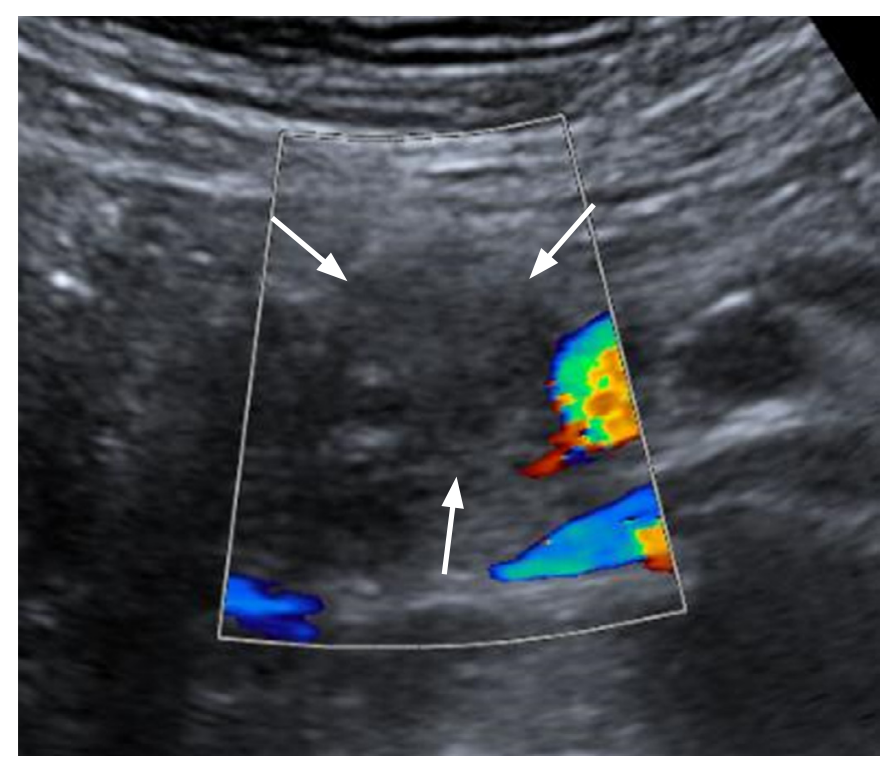

C

of the lesion in the neck/body, solid features, and a relatively large size $(15.50 \pm 10.08 \mathrm{~mm}$ vs. $23.09 \pm 12.93 \mathrm{~mm})$ were significantly associated with a higher detection rate in the univariate analysis ( $P<0.001, P=0.047$, and $P<0.001$, respectively). In the multivariable analysis, location in the neck or body (OR, 3.238; $95 \% \mathrm{Cl}, 1.926$ to 5.443) and size (OR, 1.070; $95 \% \mathrm{Cl}, 1.044$ to 1.096) were found to be significant variables $(\mathrm{P}<0.001)$.

Lesions located in the neck or body of the pancreas showed the highest detectability (76 of 102, 74.5\%) compared to those in other parts of the pancreas. US is widely used as a tool for examining intra-abdominal organs in clinical practice. However, despite its safety and cost-effectiveness, the pancreas is recognized

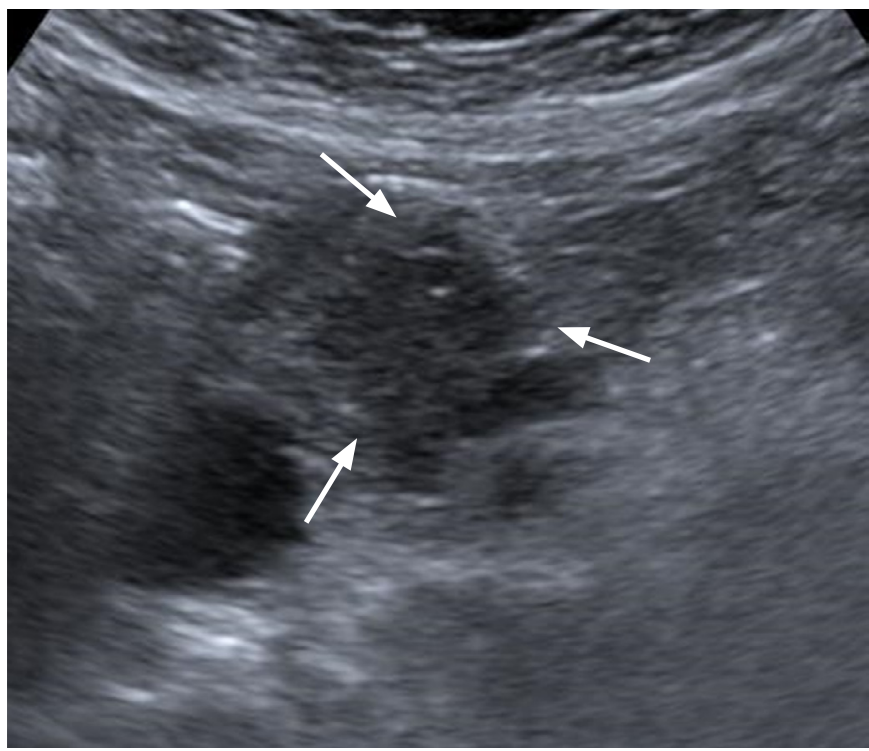

B

Fig. 4. Pancreatic cancer detected on abdominal ultrasonography (US) in a 76-year-old woman.

A. Endoscopic US shows a hypoechoic mass (arrows) in head of the pancreas with upstream main pancreatic duct (MPD) dilatation (arrowhead). B. US also shows a 2.7-cm ill-defined hypoechoic lesion in the head of the pancreas (arrows) with upstream MPD dilatation. C. A color Doppler US delineates no demonstrable vascularity within the lesion (arrows).

to be one of the most difficult organs to evaluate using US due to its deep retroperitoneal location [16]. According to previous reports $[15,16,24-27]$, US showed a varied range of diagnostic performance for the detection of pancreatic cysts. Several studies even reported that the prevalence of pancreatic cysts detected on screening US was approximately $0.1 \%$ to $0.3 \%[24,25]$, despite the rising prevalence of pancreatic cysts. In addition, while several studies reported that US showed a sensitivity of approximately $90 \%$ for detecting pancreatic tumors $[15,26,27]$, other studies reported that its sensitivity of US was no more than $70 \%$. These remarkable differences in results seem to be derived from many variables, such as lesion size, lesion location, the examiner's clinical experience, 


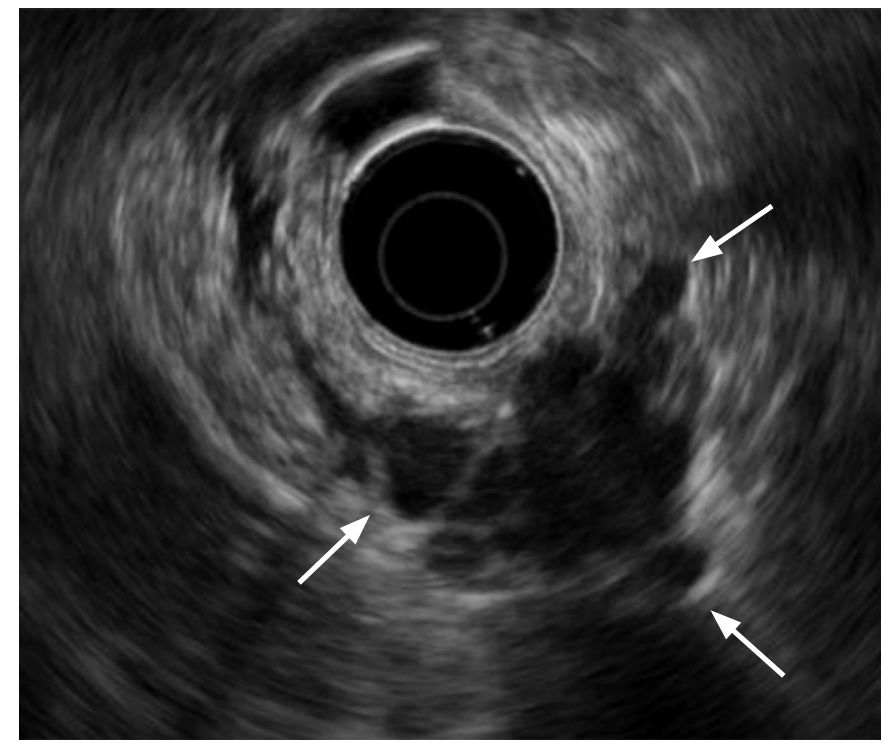

A

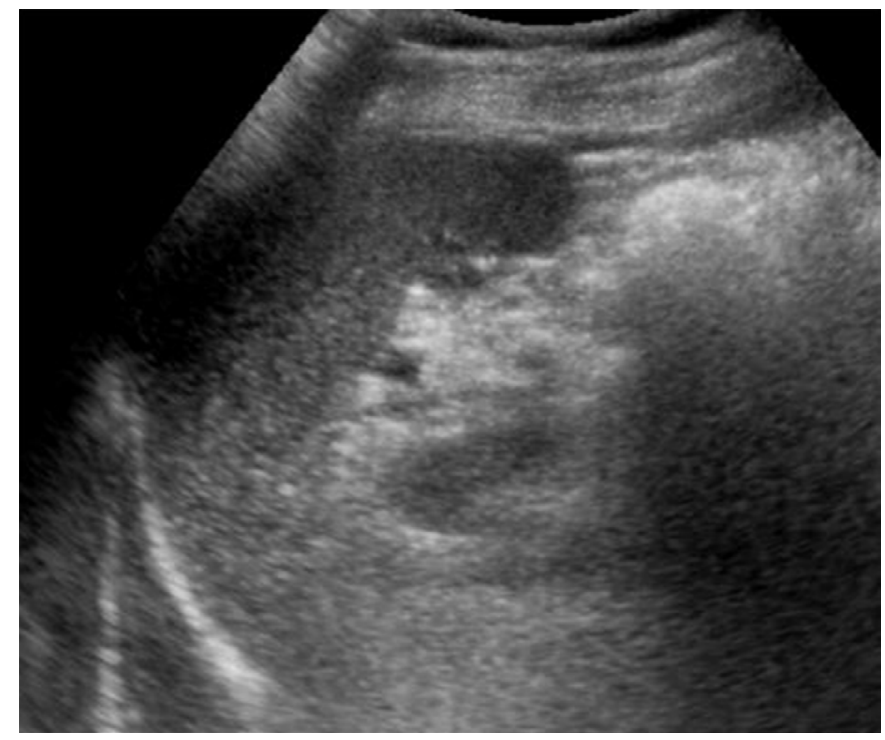

C

and the patient's condition. In our study, the overall detection rate of focal pancreatic lesions by US was $61.5 \%$ (208 of 338). The detection rate of solid lesions was significantly higher than that of cystic lesions $(70.6 \%$ and $58.5 \%$, respectively; $P=0.047)$ in the univariate analysis. To the best of our knowledge, no previous attempt has been made to compare the diagnostic performance of US according to whether a pancreatic lesion is solid or cystic. Therefore, the reason for this discrepancy in the results is uncertain. However, since solid lesions are significantly larger than cystic lesions, we surmise that lesion size could function as a confounding factor affecting the detection rate of these two types of lesions. Furthermore, the secondary changes induced by solid cystic lesions

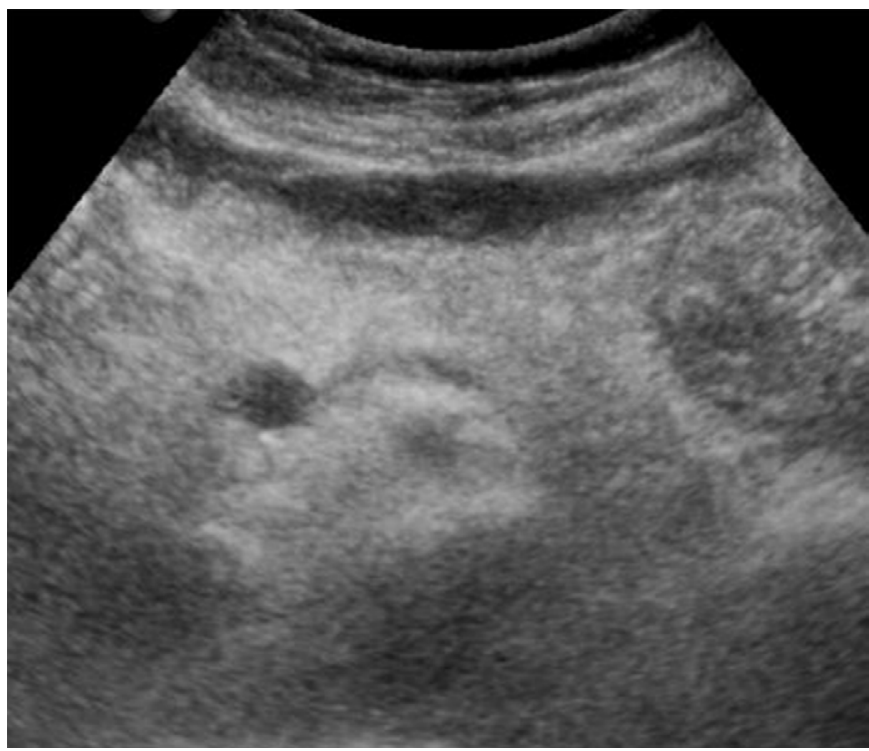

B

Fig. 5. Undetected pancreatic cyst on abdominal ultrasonography (US) in an 80-year-old woman.

A. Endoscopic US shows a 3.8-cm lobulated and septated cystic lesion in the tail of the pancreas (arrows). B. No demonstrable focal lesion was found on a follow-up US in the tail of the pancreas. C. US using the spleen as an acoustic window also failed to delineate the cystic lesion in the tail of the pancreas.

(including pancreatic cancer) in the MPD, upstream pancreatic parenchyma, or bile duct could increase the detectability of solid focal pancreatic lesions by US.

The location of focal pancreatic lesions is an important factor influencing their detectability by US. Although during US, we attempt to examine the entire pancreatic parenchyma, including the uncinate process, head, neck, body, and tail, US has inherent limitations for visualizing the entire pancreas $[15,16,28,29]$. When visualization of the pancreas is limited due to a poor sonic window, it is possible to use various scanning techniques, such as applying compression and moving the transducer. In particular, it is difficult to visualize the entire pancreatic tail even when using the spleen as an acoustic 
window. In our study, the lowest detection rate was found for lesions located in the tail $(33.8 \%)$ and the highest detection rate was found for lesions located in the neck or body $(74.5 \%)$. Clinicians should be aware of this limitation of US, and US examinations of the pancreas should be performed with great care.

Our study has several limitations. First, as this study was retrospectively designed, selection bias was inevitable and patients' body habitus was not evaluated as a variable affecting the detectability of pancreatic lesions. Second, we only included patients in whom focal pancreatic lesions were identified by EUS, and we set the EUS findings as the reference image for the focal pancreatic lesions. However, we cannot be sure that our enrolled patients represent the entire spectrum of incidental pancreatic lesions. In order to overcome these limitations, further prospective studies would be required in the future. Third, US is an operator-dependent modality. To obtain adequate images using US, the operator must have sufficient clinical experience. A poor sonic window is also one of the weaknesses of the modality, and this factor is influenced by patient-related factors such as body habitus and cooperation. Fourth, we did not limit the temporal relationship and time interval between abdominal US and EUS, and the time interval between the two examinations was quite long in some cases. In patients with pancreatic cysts, the follow-up interval was somewhat longer than that of patients with solid pancreatic lesions, which may have acted as a confounding factor.

In conclusion, US is useful for the detection and the follow-up of focal pancreatic lesions, especially for lesions located in the neck or body and for relatively large lesions.

ORCID: Seo-Youn Choi: https://orcid.org/0000-0002-2434-8779; Jung Hoon Kim: https://orcid.org/0000-0002-8090-7758; Hyo Won Eun: https://orcid.org/0000-00017785-6470; Hwaseong Ryu: https://orcid.org/0000-0003-3143-3733

\section{Author Contributions}

Conceptualization: Kim JH. Data acquisition: Kim JH, Eun HW, Ryu H. Data analysis or interpretation: Choi SY, Eun HW. Drafting of the manuscript: Choi SY, Eun HW, Ryu H. Critical revision of the manuscript: Choi SY, Kim JH. Approval of the final version of the manuscript: all authors.

\section{Conflict of Interest}

No potential conflict of interest relevant to this article was reported.

\section{References}

1. Zhang XM, Mitchell DG, Dohke M, Holland GA, Parker L. Pancreatic cysts: depiction on single-shot fast spin-echo MR images. Radiology 2002;223:547-553.

2. Lee KS, Sekhar A, Rofsky NM, Pedrosa I. Prevalence of incidental pancreatic cysts in the adult population on MR imaging. Am J Gastroenterol 2010;105:2079-2084.

3. Laffan TA, Horton KM, Klein AP, Berlanstein B, Siegelman SS, Kawamoto $S$, et al. Prevalence of unsuspected pancreatic cysts on MDCT. AJR Am J Roentgenol 2008;191:802-807.

4. Spinelli KS, Fromwiller TE, Daniel RA, Kiely JM, Nakeeb A, Komorowski RA, et al. Cystic pancreatic neoplasms: observe or operate. Ann Surg 2004;239:651-657.

5. Chernyak V, Flusberg M, Haramati LB, Rozenblit AM, Bellin E. Incidental pancreatic cystic lesions: is there a relationship with the development of pancreatic adenocarcinoma and all-cause mortality? Radiology 2015;274:161-169.

6. Tanaka M, Fernandez-del Castillo C, Adsay V, Chari S, Falconi M, Jang JY, et al. International consensus guidelines 2012 for the management of IPMN and MCN of the pancreas. Pancreatology 2012;12:183-197.

7. Allen PJ, D'Angelica M, Gonen M, Jaques DP, Coit DG, Jarnagin WR, et al. A selective approach to the resection of cystic lesions of the pancreas: results from 539 consecutive patients. Ann Surg 2006;244:572-582.

8. Lahav M, Maor Y, Avidan B, Novis B, Bar-Meir S. Nonsurgical management of asymptomatic incidental pancreatic cysts. Clin Gastroenterol Hepatol 2007;5:813-817.

9. Uehara $H$, Nakaizumi A, Ishikawa 0 , lishi H, Tatsumi K, Takakura $R$, et al. Development of ductal carcinoma of the pancreas during follow-up of branch duct intraductal papillary mucinous neoplasm of the pancreas. Gut 2008;57:1561-1565.

10. Tada M, Kawabe T, Arizumi M, Togawa O, Matsubara S, Yamamoto $\mathrm{N}$, et al. Pancreatic cancer in patients with pancreatic cystic lesions: a prospective study in 197 patients. Clin Gastroenterol Hepatol 2006; 4:1265-1270.

11. Bardeesy N, DePinho RA. Pancreatic cancer biology and genetics. Nat Rev Cancer 2002;2:897-909.

12. Rickes S, Unkrodt K, Neye H, Ocran KW, Wermke W. Differentiation of pancreatic tumours by conventional ultrasound, unenhanced and echo-enhanced power Doppler sonography. Scand I Gastroenterol 2002;37:1313-1320.

13. Miura F, Takada T, Amano H, Yoshida M, Furui S, Takeshita K. Diagnosis of pancreatic cancer. HPB (Oxford) 2006;8:337-342.

14. Yu MH, Lee JY, Kim JH, Han JK, Choi BI. Value of near-isovoxel ultrasound for evaluation of ductal communications with pancreatic cystic lesions: correlation with magnetic resonance cholangiopancreatography. Ultrasound Med Biol 2013;39:22792284.

15. Jeon JH, Kim JH, Joo I, Lee S, Choi SY, Han JK. Transabdominal ultrasound detection of pancreatic cysts incidentally detected 
at CT, MRI, or endoscopic ultrasound. AJR Am J Roentgenol 2018;210:518-525.

16. Martinez-Noguera A, D'Onofrio M. Ultrasonography of the pancreas. 1. Conventional imaging. Abdom Imaging 2007;32:136149.

17. Kamata K, Kitano M, Kudo M, Sakamoto H, Kadosaka K, Miyata $T$, et al. Value of EUS in early detection of pancreatic ductal adenocarcinomas in patients with intraductal papillary mucinous neoplasms. Endoscopy 2014;46:22-29.

18. Ahmad NA, Kochman ML, Lewis JD, Ginsberg GG. Can EUS alone differentiate between malignant and benign cystic lesions of the pancreas? Am J Gastroenterol 2001;96:3295-3300.

19. Sakamoto H, Kitano M, Suetomi Y, Maekawa K, Takeyama Y, Kudo M. Utility of contrast-enhanced endoscopic ultrasonography for diagnosis of small pancreatic carcinomas. Ultrasound Med Biol 2008;34:525-532.

20. Gonzalo-Marin J, Vila JJ, Perez-Miranda M. Role of endoscopic ultrasound in the diagnosis of pancreatic cancer. World J Gastrointest Oncol 2014;6:360-368.

21. de Jong K, Nio CY, Hermans JJ, Dijkgraaf MG, Gouma DJ, van Eijck $\mathrm{CH}$, et al. High prevalence of pancreatic cysts detected by screening magnetic resonance imaging examinations. Clin Gastroenterol Hepatol 2010;8:806-811.

22. Johnson CD, Stephens DH, Charboneau JW, Carpenter HA, Welch TJ. Cystic pancreatic tumors: CT and sonographic assessment. AJR Am J Roentgenol 1988;151:1133-1138.

23. Ishikawa $H$, Hirooka Y, Itoh A, Hashimoto $S$, Okada N, Itoh T, et al.
A comparison of image quality between tissue harmonic imaging and fundamental imaging with an electronic radial scanning echoendoscope in the diagnosis of pancreatic diseases. Gastrointest Endosc 2003; 57:931-936.

24. Ikeda M, Sato T, Morozumi A, Fujino MA, Yoda Y, Ochiai M, et al. Morphologic changes in the pancreas detected by screening ultrasonography in a mass survey, with special reference to main duct dilatation, cyst formation, and calcification. Pancreas 1994;9:508-512.

25. Ishikawa T, Takeda K, Itoh M, Imaizumi T, Oguri K, Takahashi H, et al. Prevalence of pancreatic cystic lesions including intraductal papillary mucinous neoplasms in patients with end-stage renal disease on hemodialysis. Pancreas 2009;38:175-179.

26. Karlson BM, Ekbom A, Lindgren PG, Kallskog V, Rastad J. Abdominal US for diagnosis of pancreatic tumor: prospective cohort analysis. Radiology 1999;213:107-111.

27. Tanaka S, Kitamra T, Yamamoto K, Fujikawa S, Imaoka T, Nishikawa $S$, et al. Evaluation of routine sonography for early detection of pancreatic cancer. Jpn I Clin Oncol 1996;26:422-427.

28. Sumi $H$, Itoh A, Kawashima H, Ohno E, Itoh $Y$, Nakamura $Y$, et al. Preliminary study on evaluation of the pancreatic tail observable limit of transabdominal ultrasonography using a position sensor and CT-fusion image. Eur J Radiol 2014;83:1324-1331.

29. Zamboni GA, Ambrosetti MC, D'Onofrio M, Pozzi Mucelli R. Ultrasonography of the pancreas. Radiol Clin North Am 2012;50:395-406. 